\title{
Good Practice
}

\section{What is a good GP?}

\author{
SARA ARBER
}

However good a GP is at diagnosis, treatment, communication, and preventive health care, it counts for little if the patients are unable to see him or her at what they deem to be the appropriate time. The length of time patients have to wait to get an appointment with their doctor is a common complaint and has been taken up in the consultative document on primary health care. ${ }^{1}$ Despite identifying this problem, however, the consultative document does not mention any of the reasons underlying this complaint, and the recommendations that it makes are unlikely to solve the problem.

\section{Influence of receptionists}

In most general practices it is the receptionist who determines when a patient is seen because she is usually the first person that patients contact when they phone to make an appointment or attend the surgery. Her main role is to control doctors' schedules so that they can work as efficiently as possible. Many GPs, however-and this attitude is also evident in the consultative document, which refers to receptionists only in the context of some statistical information about their numbers-seem to see the receptionist as little more than a "colourless sounding board." This is unfortunate, to say the least, because her influence is considerable. Doctors need to recognise that the receptionist has a gatekeeping role. She acts not merely as a passive filter for communications but also exercises considerable judgment and discretion in deciding whether or not to speed up or, in effect, block a patient's access to the doctor.

Patients are thus dependent on receptionists to obtain services from their GPs, whether it is to talk to the doctor on the phone, a

Department of Sociology, University of Surrey, Guildford, Surrey GU2 5XH SARA ARBER, BSC, MSC, senior lecturer domiciliary visit, or an early appointment in the surgery. She is an intermediary between patients who want access and doctors who may be concerned to rationalise their contacts with patients. I believe, therefore, that there is a good argument for suggesting that the concept of the doctor-patient relationship should be extended to the doctor-receptionist-patient relationship. This would emphasise the receptionists' vital role. After all, their actions are central to the accessibility of doctors. Those who are overprotective may discourage patients from consulting the doctor, which in turn may result in unnecessary suffering and anxiety. Those who are friendly, reassuring, and sympathetic can considerably upgrade-in the patient's eyes-the quality of service that the surgery offers.

Doctors therefore have a responsibility to monitor the performance of their receptionist and ensure that she is given a clear set of rules to follow. Merely leaving her to filter their workload because the practice provides insufficient scheduled appointment sessions to cope with the demand leads to dissatisfied hostile patients, and the antagonism so generated is deflected from the doctor to the receptionist. The concept of the officious receptionist who forms a barrier between the patient and the well meaning but overworked doctor is a familiar one, but it needs to be remembered that the fault lies in the structure and organisation of the practice, and this is the doctors' responsibility.

Commonsense suggests, and our research evidence backs this up, that the actions of receptionists vary. Receptionists who act more as a filter, or who work in a practice where the appointment book is always full, tend to be much more inquisitive and intrusive in the way they question patients and in turn receive much more criticism from patients. ${ }^{2}$

Since the health needs of the public are unpredictable, varying between times of the year and days of the week, appointment systems in general practice should not be rigid. Some built in flexibility is essential so that the time lag between requesting an appointment and getting one is minimal. In theory this should not be too difficult to arrange, but one third to a half of patients still have 
difficulty getting access to their doctors, ${ }^{3+}$ and it is not unusual for patients to have to wait three days or longer for an "urgent" appointment.

\section{Appointment systems}

When assessing how well apointment systems are structured to deal with acute sickness the following points should be considered:

(1) The method of coping with patients who do not have an appointment but wish to see the doctor as soon as possible. The main ways of dealing with this are as follows: $(a)$ give the patient the next available appointment; $(b)$ have a proportion of appointments free to be filled only by patients who request an appointment that day; (c) fit the patient in between scheduled appointments; $(d)$ see the patient at the beginning or end of surgery, or both; $(e)$ the patient is seen by one specific (duty) doctor.

(2) The criteria on which a patient is classified as an "urgent" case. The most restrictive criterion would be a "medical emergency" and the least restrictive "any condition that in the patient's view warrants urgent attention."

(3) Who should make the decision that patients with a particular condition should be seen early? The alternatives include $(a)$ the patient; $(b)$ the doctor; $(c)$ a nurse; $(d)$ a receptionist or secretary.

These issues are discussed rarely in general practice yet they are crucial to any assessment of the accessibility of patients to GPs. Badly organised appointment systems may act as rationing systems, reducing the patient's access to general practitioner care.

In some practices, the system of dealing with acute sickness leads to lack of continuity of care. This is explicitly built into systems where a "duty" doctor sees unbooked patients. In other cases patients may have to see a different doctor if their own doctor does not have a surgery that day, or they may see a doctor who works faster or is less popular and hence is able to see more unbooked patients. But is continuity of care of less relevance when treating acute sickness than long term illness or preventive health, both of which can be more easily planned in advance?

\section{Defining urgency}

If GPs accept, and presumably most do, that urgent cases can be fitted in or seen at special times the next major issue is the definition of urgency. Field suggests that "Urgent is a subjective description and it could be argued that any patient who says he needs to be seen the same day has by definition an urgent problem." Cartwright and
Anderson, however, found that the definition of urgency is rarelyw made by the patient; they found that $91 \%$ of patients said the decision about how soon they could get an appointment was made $\stackrel{\Omega}{\Omega}$ by the receptionist.

Receptionists are the ones who have to put into operation whatever type of appointment system is specified by the doctors in that practice. They are in the unenviable position of either bearing the brunt of negative criticism from patients who cannot get ano appointment as soon as they want one or the wrath of doctors whom want to finish their surgery "on time" rather than having a series of. patients "fitted in" or seen after surgery hours. The receptionist is therefore subject to conflicting pressures. Whether she acts more as a "barrier" to patients is likely to be a reflection of practice policy in terms of the definition of urgent and the way of dealing with "urgent" cases.

I believe that the best way to deal with requests for urgent appointments is to let the patient uefine urgency and the recep-es tionist then arrange for them to be seen that day with the minimal$\vec{\circ}$ disruption to the doctors' daily routine. This can be achieved onlyby continually monitoring the number of patients' requests for bothw urgent and non-urgent appointments so that the appointment system may be adjusted to achieve the optimum balance. The ratio of prebooked to unbooked appointment times would probably varyin between the different days of the week and times of the year. Such a ${ }^{+}$ system should result in better organised doctors, more contentedo patients, less irate receptionists, and fewer patients who fail to seek medical care when they think they need to because of the difficultyin in obtaining an appointment.

The consultative document notes the importance of computers in응 general practice to implement call and recall systems for usew in preventive health care. Experimental schemes using microcomputers to organise flexible and efficient appointment systems should also receive support.

In conclusion, one answer to the question "What is a good GP?" is a GP who organises his or her practice so that patients can obtain $\overrightarrow{0}$ appointments easily and quickly and where they, rather than the receptionist, decide when they should see the doctor.

\section{References}

1 Secretaries of State for Social Services, Wales, Northern Ireland, and Scotland. Primary health care. an agenda for discussion. London: HMSO, 1986:14. (Cmnd 9771.)

Arber S, Sawyer L. The role of the receptionist in general practice: "a dragon behind the desk"? Soc Sci Med 1985;20:911-21.

3 Arber S, Sawyer L. Do appointment systems work? Br Med $\mathcal{F}$ 1982;283:1367-70.

4 Cartwright A, Anderson R. General practice revisited. London: Tavistock Publications, 1981.

Field J. Problems of urgent consultations within an appointment system. $\mathcal{I} R$ Coll Gen Pracr $1980 ; 30: 173-7$

\section{YEARS AGO}

Even in France the doctors are not altogether happy, although their number is said not to exceed some fifteen thousand, not an excessive supply for forty million inhabitants. There have been heard of late shrill complaints from the poorer communes of the high fees which country doctors find it necessary to charge for the long journeys which their visits often necessitate. An effort has been made to obtain permission for the pharmaciens to practise on a small scale at a reduced tariff. "It would be," says the petition, "a truly democratic work to place health within everyone's reach"-health, apparently, being taken as synonymous with medication. This is not the only solution that has been suggested, for a correspondent, writing to a French contemporary, urges that Government should "favour by every possible means the residence in each commune of a doctor or officier de santé." It is difficult to imagine what inducements government could offer to medical men to reside in communes quite incapable of maintaining them, short of providing a subsidy. Even then the number of doctors would require to be increased to 36,000 , and putting their income at the modest figure of $£ 150$, the cost would not be less than $£ 3,000,000$ per annum. As it is, a very large number of country doctors do not make an income of more than from $£ 100$ to $£ 300$ a year, a consideration which may cheer our own provincial practitioners who think themselves ill rewarded with $£ 500$. In France, moreover, there is no poor-law medical attendance to relieve the strain, and medical clubs exist in quite an embryonic condition. The suggestion with respect to the local $N$ chemists would be inadequate. They already deal largely in prescribing overw the counter, and if they took upon themselves the functions of the medicalo man, the same difficulty in providing for the cost of the long journeys would hold good. One reason why the income of provincial medical men is so miserably small in many instances is that the parsimonious peasant resorts to the bone-setter and the herbalist for minor ailments, and only sends for the doctor when the patient is in extremis, just to avoid trouble about the registration of death. Numerous other suggestions have been made, all more $\overrightarrow{\mathbb{C}}$ or less inappropriate to the end in view. For instance, it is urged that medical $\stackrel{\varrho}{\square}$ men should have free passes on the local railways, and should be exempted from the patente, a tax which is paid for permission to practise any trade oro profession. The State is advised to enforce more rigorously the law against the illegal practice of medicine, and to make the doctor's bill a preferentialo claim, not amenable to any statute of limitations. But the only means by which poor people can ensure medical attendance when sick is cooperation. (British Medical Fournal 1887;ii:195.) 